\title{
Development of the VX2 Pancreatic Cancer Model in Rabbits: A Platform to Test Future Interventional Radiology Therapies
}

\author{
Aaron C. Eifler, BS, Robert J. Lewandowski, MD, Sumeet Virmani, MD, Johnathan C. \\ Chung, MD, Dingxin Wang, MS, Richard L. Tang, MD, Barbara Szolc-Kowalska, MD, Gayle \\ E. Woloschak, PhD, Guang-Yu Yang, MD, PhD, Robert K. Ryu, MD, Riad Salem, MD, MBA, \\ Andrew C. Larson, PhD, Eric Cheon, MD, Matthew Strouch, MD, David J. Bentrem, MD, and \\ Reed A. Omary, MD, MS \\ Department of Radiology (A.C.E, R.J.L., S.V., J.C.C., D.W., R.L.T., M.F.M, R.K.R., R.S., A.C.L., \\ R.A.O.), Department of Radiation Oncology (B.S.K., G.E.W.), Department of Surgery (E.C., M.S., \\ D.Z., D.J.B), Department of Biomedical Engineering (D.W., A.C.L., R.A.O.), Department of \\ Pathology (G-Y,Y), Feinberg School of Medicine and Robert H. Lurie Comprehensive Cancer \\ Center (M.F.M, G.E.W., D.J.B., A.C.L., R.A.O.), Northwestern University, 737 N Michigan Ave, \\ Ste 1600, Chicago, IL 60611
}

\begin{abstract}
PURPOSE-An animal model of pancreatic cancer that is large enough to permit imaging and catheterization would be desirable for interventional radiologists to develop novel therapies for pancreatic cancer. The purpose of this study was to test the hypothesis that the VX2 rabbit model of pancreatic cancer could be developed as a suitable platform to test future interventional therapies.
\end{abstract}

\begin{abstract}
MATERIALS AND METHODS-The authors implanted and grew three pancreatic VX2 tumors per rabbit in six rabbits. Magnetic resonance (MR) imaging was performed at two weeks to confirm tumor growth. At three weeks, the authors selectively catheterized the gastroduodenal artery under guidance of x-ray digital subtraction angiography (DSA). T2-weighted anatomic, diffusion-weighted (DWI), and transcatheter intraarterial perfusion (TRIP) MR imaging were then performed. Following imaging, tumors were confirmed at necropsy and histopathology. Size of tumors at two and three weeks was compared using a paired $t$-test $(\mathrm{P}=.05)$.
\end{abstract}

\begin{abstract}
RESULTS-VX2 pancreatic tumors were grown in $6 / 6$ rabbits. The difference between tumor size at two and three weeks, $1.29 \mathrm{~cm}( \pm 0.39)$ and $1.91 \mathrm{~cm}( \pm 0.50)$ respectively, was statistically significant $(\mathrm{p}<.001)$. All tumors were confirmed to be located within pancreatic tissue via histopathology. DSA, TRIP MR, DWI and anatomic MR imaging was successful in 5/6 rabbits. DWI and anatomic MR imaging was successful in $6 / 6$ rabbits.
\end{abstract}

CONCLUSION-The VX2 rabbit model of pancreatic cancer is feasible, as verified by imaging and pathologic correlation, and may be a suitable platform to test future interventional therapies.

\section{INTRODUCTION}

As the fourth leading cause of cancer related mortality in the United States (1), pancreatic cancer carries with it a dismal prognosis. Nearly $99 \%$ of those diagnosed with pancreatic

address of correspondence to R.A.O.; reed@ northwestern.edu.

None of the authors has identified a conflict of interest.

At time of original submission (8/2008), this material had not been presented at an SIR Annual Meeting. Since then, it has been presented as a poster at the 2009 SIR Annual Meeting (winning the SIR Poster Award). 
cancer will die of their disease, with median survival of 6 months and 5-year survival of less than 5\% across all stages (1-4).

Despite recent advances in understanding of molecular and genetic mechanisms of the disease, pancreatic cancer remains resistant to nearly all available treatments (5) with surgical resection remaining the only potentially curative measure $(6,7)$. Resection, however, is possible in less than $20 \%$ of cases and of those patients, median 5-year survival is only $12 \%$ (8). Mortality in unresectable cases is practically guaranteed and although future therapies aimed at recently elucidated molecular pathways of the disease may offer hope for more effective palliation, traditional chemotherapy combinations are yielding only slight gains (9).

Given these grim statistics, there is a clear need for innovative approaches to treat pancreatic cancer. In the past decade, novel interventional radiology therapies directed towards hepatic malignancies, such as chemoembolization, radioembolization, and radiofrequency ablation, have gained widespread acceptance because of their ability to improve survival and/or induce imaging response. We propose that similar improvements for patients suffering from pancreatic cancer may be feasible. In fact, preliminary studies of arterial infusion chemotherapy for advanced pancreatic cancer have shown that this therapy is not only possible (10-12), but also may provide significant gains in 1-year survival (13). Thus, we postulate that novel interventional radiology therapies for pancreatic cancer may be developed that could potentially offer significant survival benefit over current systemic therapies. An acceptable pre-clinical model to test such therapies in animals large enough to permit catheterization would be desirable.

The VX2 model of cancer in rabbits has been established previously in translational studies of uterine $(14)$, liver $(15,16)$, lung $(17,18)$, brain $(19,20)$ and renal $(21,22)$ neoplasms. The purpose of this study was to test the hypothesis that the VX2 rabbit model of pancreatic cancer could be developed as a suitable platform to test future interventional therapies. Such a platform requires the ability to grow tumors that can be a) depicted with magnetic resonance (MR) imaging; b) targeted with $x$-ray digital subtraction angiography (DSA), and c) correlated with pathology.

\section{MATERIALS AND METHODS}

\section{VX2 Animal Model}

Our Animal Care and Use Committee approved all experiments. VX2 tumors were produced in the pancreata of six New Zealand White rabbits each weighing about 3-4 kg using the chunk implantation method due to its demonstrated high efficacy (23). There are no known risks of VX2 infection in humans. We used two hind limb tumor (donor) rabbits to propagate and maintain the VX2 strain. After euthanasia, one of these hindlimb rabbits was used to study rabbit pancreatic anatomy prior to implantation.

For all experiments, the VX2 tumor cell solution was prepared with the following technique. A VX2 tumor was rinsed in Ham's F-10 Nutrient Mixture with L-glutamine (GIBCO; Invitrogen, Carlsbad, Calif) and homogenized into a cell suspension, which was then filtered through a $40-\mu \mathrm{m}$ mesh cell strainer (BD Biosciences, San Jose, Calif). Cells were pelleted by centrifugation at $500 \mathrm{~g}$ for 5 minutes. The supernatant was discarded and the cell pellet was re-suspended in equal volume of basic methylcellulose media (StemCell Technologies, British Columbia, Canada). The VX2 tumor cell solution then was injected into donor rabbit hind limbs and allowed to develop for 3-4 weeks. 
All implantation surgeries were performed by an attending surgical oncologist, with the assistance of animal technologists. VX2 tumors were freshly harvested from donor hind limb rabbits shortly before surgery and cut into $1 \mathrm{~mm}^{3}$ fragments which were placed on ice until time of implantation. In a mini-laparotomy, the recipient rabbit pancreas was exposed and a small stab wound made in the pancreas parenchyma with a number 11 knife blade. We then inserted the tumor piece deeply into the stab wound. To seal the wound and to prevent the tumor fragment from getting dislodged, a $1 \mathrm{~cm}^{2}$ piece of Surgicel (Ethicon, Johnson \& Johnson, Somerville, New Jersey) was placed onto the incision site (Fig 1). We repeated this process two more times, approximately $2-4 \mathrm{~cm}$ apart, for a total of three tumor fragment implantations in each of the 6 recipient rabbits. The abdomen was then closed in three layers and the tumors allowed to incubate for two weeks for initial check up imaging and three weeks for final imaging. A literature search and abdominal dissection of the two hind limb donor rabbits prior to implantation surgery were used to clarify rabbit pancreatic anatomy.

For all surgeries and imaging studies, each rabbit was anesthetized with an intramuscular injection of $44 \mathrm{mg} / \mathrm{kg}$ ketamine hydrochloride (Ketaset; Fort Dodge Laboratories, Fort Dodge, Iowa) and 3-5 mg/kg xylazine (Xyla-ject; Phoenix Pharmaceuticals, St Joseph, Mo). In addition, we administered 2\%-3\% isoflurane (Iso-Flo; Abbott Laboratories, North Chicago, Ill) via a mask during surgery. Rabbits were intubated with a 3-F endotracheal tube, and inhalational 2\%-3\% isoflurane was administered by using a small animal ventilator (Harvard Apparatus, Holliston, Mass). Additional intramuscular administration of $22 \mathrm{mg} / \mathrm{kg}$ ketamine was performed as needed.

\section{Tumor Progression Imaging}

At two weeks following pancreatic VX2 implantation, MR images were obtained by using a 1.5-T scanner (Espree; Siemens Medical Solutions, Erlangen, Germany). Rabbits were imaged in the supine position with a conventional single-channel head coil. We obtained T2weighted turbo spin echo images with the following parameters: 4210/86 (repetition time msec/echo time msec), $3 \mathrm{~mm}$ thick sections, $180 \times 121 \mathrm{~mm}^{2}$ field of view, $256 \times 172$ matrix, $150^{\circ}$ flip angle, $205 \mathrm{~Hz}$ per pixel bandwidth, and two signals acquired. Tumor growth was considered positive when tumor was identified in axial and coronal imaging planes by two independent attending interventional radiologists.

\section{DSA and Catheter Placement}

We performed x-ray DSA by using a C-arm unit (Powermobil; Siemens Medical Solutions) at three weeks after implantation. All catheterization procedures were performed by two attending interventional radiologists who specialize in transcatheter interventional oncology procedures. The femoral artery was accessed through a surgical cut-down and catheterized with a 22-gauge angiocatheter (Terumo Medical, Somerset, NJ). A 2-F catheter (Cook, Bloomington, IN) was advanced over a 0.014 -inch diameter guide wire (Terumo Medical). We then selectively catheterized the gastroduodenal artery. We performed DSA of the celiac artery and gastroduodenal artery with hand injections of iohexol (Omnipaque 350;

Amersham Health, Princeton, NJ). Once catheter placement was complete, 2-0 silk suture in the rabbit's groin was used to hold the catheter in place while the rabbit was transferred to the adjacent MR suite.

\section{MR Imaging Following Catheterization}

MR imaging after catheter placement was performed using the same Siemens 1.5-T Espree clinical MR scanner. Rabbits underwent imaging with a clinical head coil in supine position. To depict tumor anatomy, we first acquired two-dimensional turbo spin-echo T2-weighted images with the following scan parameters: repetition time/echo time, 4210/86 msec; 205 
Hz/pixel bandwidth; flip angle of $150^{\circ} ; 256 \times 172$ matrix; slice thickness of $3 \mathrm{~mm}$; and field of view of $180 \times 121 \mathrm{~mm}^{2}$.

Diffusion weighted MR imaging was performed with single-shot spin-echo echo planar imaging with the following scan parameters: repetition time/echo time, $4000 / 93 \mathrm{msec}$; slice thickness $3 \mathrm{~mm}$; bandwidth, $1185 \mathrm{~Hz} /$ pixel; partial Fourier factor, 6/8; nonselective fat saturation; twice refocused spin-echo diffusion weighting to reduce eddy current-induced distortion with $\mathrm{b}$ values of 0,50 and $500 \mathrm{sec} / \mathrm{mm}^{2}$. The purpose of diffusion weighted imaging was to provide functional assessment of water mobility within the tumor, with necrotic regions of tumor expected to show increased water mobility compared to viable regions (24).

To depict tumor perfusion, we used a transcatheter intraarterial perfusion (TRIP) MR imaging technique (25) that incorporated a three-dimensional spoiled gradient echo sequence with the following parameters: repetition time/echo time, $5 / 1.6 \mathrm{msec} ; 15^{\circ}$ flip angle; contiguous axial slices of $3 \mathrm{~mm}$ thickness; eight partitions; $200 \times 100 \mathrm{~mm}^{2}$ field of view; $128 \times 64$ matrix; and $660 \mathrm{~Hz} /$ pixel bandwidth. This TRIP MR imaging sequence is a real-time three-dimensional MR fluoroscopy technique (26) that rapidly and continuously acquires images covering the entire tumor volume during transcatheter contrast medium injection. TRIP MR imaging scans were obtained during hand injections of $2 \mathrm{~mL} \mathrm{20 \%}$ gadopentetate dimeglumine solution (Magnevist; Berlex, Wayne, NJ) over a period of 5 seconds via catheter previously placed during DSA. Each contrast medium injection was immediately followed by a 4-mL saline solution flush injected over a period of 5 seconds. For each TRIP MR imaging scan, the images covering the entire pancreas were continuously sampled at 1.6-second intervals for 100 seconds.

Before and after TRIP MR imaging, we obtained anatomic images with two-dimensional T1-weighted gradient-echo MR imaging. The T1-weighted scan parameters were as follows: repetition time/echo time, $193 / 6 \mathrm{msec}$; average of 2; flip angle of $80^{\circ}$; bandwidth of $475 \mathrm{~Hz} /$ pixel; slice thickness of 3; $256 \times 160$ matrix; and field of view of $180 \times 113 \mathrm{~mm}^{2}$.

\section{Necropsy and Histopathology}

Once MR imaging was completed, each rabbit was sacrificed with an intravenous administration of 150-200 mg/kg sodium pentobarbital (Euthasol; Delmarva Laboratories, Midlothian, VA). Necropsy was performed in each recipient rabbit and the tumor was harvested for pathologic confirmation of VX2 tumor growth and location in pancreatic tissue. Pancreatic tumors were inspected grossly to verify anatomic consistency with MR images. Tumor samples, including surrounding tissue, were then removed, embedded in paraffin, and mounted on glass slides. These $4-\mu \mathrm{m}$-thick slices were stained with hematoxylin and eosin. Histopathologic analysis was performed by an attending surgical pathologist with 14 years of specialization in pancreatic cancer.

\section{Image Analysis}

MR images and DSA were reviewed in conjunction with one another. MR images were analyzed on a dedicated computer workstation (Argus; Siemens Medical Solutions). Unenhanced and contrast-enhanced MR images were evaluated in consensus by two attending interventional radiologists. Tumor diameters were measured on axial MR images. Diffusion-weighted and TRIP MR images were analyzed qualitatively to assess intratumoral differences in water mobility and tumor perfusion, respectively. 


\section{Statistical Analysis}

Mean ( \pm standard deviation) tumor diameters were calculated. The difference between the diameter at two and three weeks was compared using a two-tailed paired $t$-test $(\alpha=.05)$ to assess growth. All statistical tests were performed with software (InStat version 3.06 for Windows; GraphPad Software, San Diego, Calif).

\section{RESULTS}

\section{VX2 Tumor Model}

We successfully grew, catheterized, and imaged pancreatic VX2 tumors in all six rabbits. Figure 1 illustrates a representative VX2 tumor implantation into the rabbit pancreas. In those six rabbits, 11 total tumors grew. Mean tumor diameter was $1.29 \mathrm{~cm}( \pm 0.39)$ at two weeks and $1.91 \mathrm{~cm}( \pm 0.50)$ at three weeks $(\mathrm{p}<.001)$. Figure 2 shows a histogram of tumor sizes at 3 weeks showing a seemingly multimodal distribution. This suggests that in some cases, two implanted tumor pieces grew together to form one larger tumor. During the 3 weeks of tumor incubation, all six rabbits remained asymptomatic.

\section{Rabbit Pancreatic Anatomy and DSA}

The rabbit pancreas was encircled by the duodenal C-loop, which was located within the peritoneal cavity and not retroperitoneally (Fig 3 ). The pancreas consisted of diffuse exocrine and endocrine glandular tissue positioned within the mesentery of the duodenum.

DSA was successfully completed in 5 of 6 rabbits. DSA showed, and gross dissection verified, that the gastroduodenal artery (GDA) supplied our pancreatic tumors (Fig 3). This finding is supported by the tumor blush seen in Figure $3 \mathrm{c}$ which resulted from iodinated contrast injected into the GDA. The GDA, like in humans, was found to branch from the common hepatic artery (CHA) and the CHA from the celiac artery. The pancreaticoduodenal arcade (PDA) originated from the GDA to supply the implanted tumor. We did not visualize the dorsal pancreatic artery or the pancreatic magna. The GDA in 1 of 6 rabbits could not be catheterized due to arterial dissection during DSA.

\section{Histopathology}

At necropsy, tumors in all six rabbits were confirmed at gross pathology. Through histopathologic examination, all masses were confirmed to be neoplastic and located within pancreatic tissue as confirmed by an attending surgical pathologist with specialization in gastrointestinal oncology. Abdominal metastases were noted among rabbits in patterns varying from no metastases to extensive seeding of the liver surface with no single pattern dominating. Figure 4 shows the typical arrangement of viable VX2 cells of the tumor periphery adjacent to asymptomatic pancreatic tissue. A band of irritated pancreatitis-like tissue can be seen between the healthy pancreatic tissue and tumor cells. VX2 tumors contained necrotic tissue predominantly within the tumor core, with viable VX2 cells located in the periphery of the tumor (Fig 4b).

\section{MR Imaging}

Tumors in all 6 rabbits were successfully depicted using anatomic and diffusion-weighted MR imaging. Anatomic T2-weighted images showed increased signal intensity within the tumors compared to surrounding tissues in locations corresponding to the abdominal right upper quadrant (Fig 5). Representative diffusion-weighted images are shown in Figure 6. In these images, the tumor shows regions of low water mobility within the viable periphery signified by the increased signal intensity (SI) at higher b values. This finding suggests increased cellularity compared to the tumor core and surrounding tissues. 
TRIP MR imaging was successful in the 5 of 6 rabbits that could be catheterized under DSA. TRIP MR scans showed tumor perfusion to the viable portions of tumor, with lack of enhancement in the necrotic portions of the tumor as shown in Figure 6. TRIP MR imaging following contrast injection into the GDA confirmed that our pancreatic tumors were supplied by the GDA, as initially found in DSA. When comparing diffusion weighted and TRIP MR imaging, regions of relatively low water mobility correlated with relatively greater tissue perfusion.

\section{DISCUSSION}

In this study, we showed that VX2 tumors could be successfully grown in the rabbit pancreas, depicted with MR imaging, and correlated with histopathology. We also showed that the arterial blood supply to the tumors could be catheterized for DSA. All six tested rabbits grew pancreatic tumors that could be detected using a) DSA, b) conventional anatomic T2-weighted MR imaging, and c) functional diffusion-weighted and TRIP MR imaging. These results suggest that the VX2 model of pancreatic cancer would be a suitable platform to test future interventional therapies.

The development of animal models of pancreatic cancer has been recognized as a highly significant unmet need (27). Rodent models that can potentially mimic the molecular biology of human pancreatic cancer include xenographic cell transplantation, carcinogen administration, and genetically engineered models (28). However, these models have not been fully proven and their suitability as appropriate models for the human pancreas has been questioned (29). Also, as their small size precludes vascular catheterization and conventional MR imaging, rodents are unable to accommodate the imaging and catheterization required to test future proposed interventional therapies. However, the larger size of our experimental New Zealand white rabbits meets the requirements to test such IR therapies.

Although we achieved VX2 tumor growth in 6/6 rabbits, because of consolidation of adjacent tumors, it was unclear how many implanted tumor pieces constituted one mature pancreatic tumor. Previous success rates for VX2 tumor growth in rabbit livers using a chunk implantation method were reported as $95 \%$ and $84 \%$ by Chen et al (30) and Virmani et al (23) respectively. Unlike the relatively dense and structured rabbit liver, the pliable rabbit pancreas may shift upon reinsertion into the abdominal cavity following surgery reducing the original 2-4 cm separation of tumor chunks achieved at implantation (Fig 1b). This closer proximity could have caused two (or more) separate tumor pieces to grow together into one mature tumor. This would explain the roughly bimodal distribution of Figure 2. Single piece tumors likely fell within the $1.5-2 \mathrm{~cm}$ range as also reported by Virmani et al (23), while the largest tumors (those $>2.5 \mathrm{~cm}$ in diameter) were most likely conglomerates of more than one implanted tumor piece. Thus, surgical implantation remains an effective method to grow VX2 tumors.

For interventional radiologists, there are several important advantages to this model. First, New Zealand white rabbits are large enough to develop and test therapies that could be delivered locally to pancreatic tumors. This method of drug delivery has been preliminarily attempted and has achieved encouraging results (13). Further exploration of this method using the model presented in this study could yield advances in pancreatic cancer therapy. Second, the VX2 tumors grown in this model are large enough to be imaged using clinical $1.5 \mathrm{~T}$ scanners. This eliminates the need for a dedicated high field scanner and permits use of existing imaging techniques. Smaller rodent models are at a disadvantage for these two reasons. Third, there are already extensive data available regarding VX2 tumors used in this study. These tumors are well characterized and the method of their induction has been 
thoroughly studied. Finally, the VX2 molecular biology includes alterations within the function of p53 (31), which also occurs in up to 75\% of pancreatic cancers (5). This suggests a degree of molecular homology between the VX2 tumors and human pancreatic tumors, further supporting this model's utility.

Given these advantages along with the relative lack of effective treatments for pancreatic cancer as discussed above, we think that the presented model could serve a vitally important role in the development of new interventional radiology therapies for pancreatic cancer and help facilitate their translation into humans. Analogous to the use of VX2 models in the pioneering of therapies for hepatocellular carcinoma (32-34), this model could be used to open pancreatic cancer to treatment by interventional radiologists using cutting edge, yet untested technologies. For example, targeted molecular therapy using functionalized gold nanoparticles aimed at anti-apoptotic proteins expressed in various forms of cancer have shown efficacy in vitro (35). Compared to conventional chemotherapeutic or embolic agents, novel approaches using nanoparticles, immunotherapy (36), or other cellular therapies (37) would be much less likely to induce caustic or ischemic injury to tissues within the vascular distribution of the pancreas, such as the duodenum. If interventional radiologists wish to participate in the development of such innovative therapies and continue the field's central role as a speciality that disrupts conventional treatment paradigms, then it is imperative to develop relevant animal models.

Our study had several important limitations. Firstly, the pathogenesis and behavior of pancreatic ductal adenocarcinoma and VX2-based tumors differ. However, this discrepancy in tumor biology is also present between VX2 carcinoma and HCC. This difference has not prevented interventional radiologists from widely employing VX2 as a model for human liver cancer. Secondly, the smaller diameter of rabbit pancreatic arteries precluded direct catheterization of the PDA, dorsal pancreatic artery, caudal pancreatic artery, and pancreata magna. In humans, these arteries all supply the pancreas. In our study, we identified the PDA, but did not visualize these other pancreatic arteries that can potentially be seen in humans. Despite the lack of direct catheterization of the PDA, TRIP MR imaging verified that targeted delivery of contrast material to the pancreatic tumors could still be achieved via catheterization of the rabbit GDA. Third, this study was not designed to address the venous and lymphatic anatomy of the rabbit pancreas. This anatomy could be the subjective of future investigation. Finally, compared to the size of the surrounding pancreas, our tumors at 3 weeks were relatively larger than the ratio of tumor size to pancreas size that might be seen clinically. This was due to the growth pattern of VX2 tumors and their typical large necrotic core. We postulate that this size discrepancy could be circumvented in the future by implanting smaller pieces of VX2 tumor.

In conclusion, we have shown that the VX2 rabbit model of pancreatic cancer is feasible, as verified by imaging and pathologic correlation. Tumors can be induced by surgical implantation, then imaged using a $1.5 \mathrm{~T}$ clinical MRI scanner with anatomic and functional techniques. Because the proximal arterial supply to the tumor can be catheterized and DSA successfully performed, it is also possible to use this model as a platform to test future transcatheter therapies. In subsequent studies, we intend to apply this model to test novel imaging techniques and therapies for pancreatic cancer.

\section{Acknowledgments}

A.C.E. was supported by the Medical Student Summer Research Program - Feinberg School of Medicine; R.J.L. was supported in part by the Society of Interventional Radiology Foundation Pilot Research Grant Program 


\section{BIBLIOGRAPHY}

1. American Cancer Society. Cancer Facts \& Figures 2008. Atlanta: American Cancer Society; 2008.

2. Ries, L.; Melbert, D.; Krapcho, M., et al. SEER Cancer Statistics Review, 1975-2005. Bethesda: National Cancer Institute; 2008.

3. Mayer, RJ. Neoplastic Disorders. In: Kasper, DL.; Fauci, AS.; Longo, DL.; Braunwald, E.; Hanser, SL.; Jameson, JL., editors. Harrison's Principles of Internal Medicine. 16. New York: McGaw-Hill; 2005. p. 537-539.

4. Carpelan-Holmstrom M, Nordling S, Pukkala E, et al. Does anyone survive pancreatic ductal adenocarcinoma? A nationwide study re-evaluating the data of the Finnish Cancer Registry. Gut 2005;54:385-387. [PubMed: 15710987]

5. Feldmann G, Maitra A. Molecular genetics of pancreatic ductal adenocarcinomas and recent implications for translational efforts. J Mol Diagn 2008;10:111-122. [PubMed: 18258927]

6. Conlon KC, Klimstra DS, Brennan MF. Long-term survival after curative resection for pancreatic ductal adenocarcinoma. Clinicopathologic analysis of 5-year survivors. Ann Surg 1996;223:273279. [PubMed: 8604907]

7. Ghaneh P, Costello E, Neoptolemos JP. Biology and management of pancreatic cancer. Gut 2007;56:1134-1152. [PubMed: 17625148]

8. Garcea G, Dennison AR, Pattenden CJ, Neal CP, Sutton CD, Berry DP. Survival following curative resection for pancreatic ductal adenocarcinoma. A systematic review of the literature. JOP 2008;9:99-132. [PubMed: 18326920]

9. Stathopoulos GP, Androulakis N, Souglakos J, Stathopoulos J, Georgoulias V. Present treatment and future expectations in advanced pancreatic cancer. Anticancer Res 2008;28:1303-1308. [PubMed: 18505070]

10. Homma H, Doi T, Mezawa S, et al. A novel arterial infusion chemotherapy for the treatment of patients with advanced pancreatic carcinoma after vascular supply distribution via superselective embolization. Cancer 2000;89:303-313. [PubMed: 10918160]

11. Ohigashi $\mathrm{H}$, Ishikawa $\mathrm{O}$, Yokayama $\mathrm{S}$, et al. Intra-arterial infusion chemotherapy with angiotensinII for locally advanced and nonresectable pancreatic adenocarcinoma: further evaluation and prognostic implications. Ann Surg Oncol 2003;10:927-934. [PubMed: 14527913]

12. Tanaka T, Sakaguchi H, Anai H, et al. Catheter position for adequate intra-arterial chemotherapy for advanced pancreatic cancer: evaluation with CT during arterial injection of contrast material. J Vasc Interv Radiol 2004;15:1089-1097. [PubMed: 15466795]

13. Miyanishi K, Ishiwatari H, Hayashi T, et al. A Phase I trial of arterial infusion chemotherapy with gemcitabine and 5-fluorouracil for unresectable advanced pancreatic cancer after vascular supply distribution via superselective embolization. Jpn J Clin Oncol 2008;38:268-274. [PubMed: 18375446]

14. Rhee TK, Ryu RK, Bangash AK, et al. Rabbit VX2 Tumors as an Animal Model of Uterine Fibroids and for Uterine Artery Embolization. J Vasc Interv Radiol 2007;18:411-418. [PubMed: 17377188]

15. Geschwind JF, Artemov D, Abraham S, et al. Chemoembolization of liver tumor in a rabbit model: assessment of tumor cell death with diffusion-weighted MR imaging and histologic analysis. J Vasc Interv Radiol 2000;11:1245-1255. [PubMed: 11099235]

16. Nijsen JF, Seppenwoolde JH, Havenith T, Bos C, Bakker CJ, van het Schip AD. Liver tumors: MR imaging of radioactive holmium microspheres--phantom and rabbit study. Radiology 2004;231:491-499. [PubMed: 15031432]

17. Goldberg SN, Gazelle GS, Compton CC, Mueller PR, McLoud TC. Radio-frequency tissue ablation of VX2 tumor nodules in the rabbit lung. Acad Radiol 1996;3:929-935. [PubMed: 8959183]

18. Ogilvie RW, Blanding JD Jr, Wood ML, Knisely WH. The Arterial Supply to Experimental Metastatic Vx2 and Xy Tumors in Rabbit Lungs. Cancer Res 1964;24:1418-1431. [PubMed: 14221798]

19. Brem SS, Zagzag D, Tsanaclis AM, Gately S, Elkouby MP, Brien SE. Inhibition of angiogenesis and tumor growth in the brain. Suppression of endothelial cell turnover by penicillamine and the 
depletion of copper, an angiogenic cofactor. Am J Pathol 1990;137:1121-1142. [PubMed: 1700617]

20. Frank JA, Girton M, Dwyer AJ, et al. A reproducible model of metastatic brain and ocular tumor by hematogenous inoculation of the VX2 tumor in rabbits. J Neurosurg 1987;67:106-109. [PubMed: 3598658]

21. Horkan C, Ahmed M, Liu Z, et al. Radiofrequency ablation: Effect of pharmacologic modulation of hepatic and renal blood flow on coagulation diameter in a VX2 tumor model. J Vasc Interv Radiol 2004;15:269-274. [PubMed: 15028812]

22. Miao Y, Ni Y, Bosmans H, et al. Radiofrequency ablation for eradication of renal tumor in a rabbit model by using a cooled-tip electrode technique. Ann Surg Oncol 2001;8:651-657. [PubMed: 11569780]

23. Virmani S, Harris KR, Szolc-Kowalska B, et al. Comparison of two different methods for inoculating VX2 tumors in rabbit livers and hind limbs. J Vasc Interv Radiol 2008;19:931-936. [PubMed: 18503910]

24. Deng J, Rhee TK, Sato KT, et al. In vivo diffusion-weighted imaging of liver tumor necrosis in the VX2 rabbit model at 1.5 Tesla. Invest Radiol 2006;41:410-414. [PubMed: 16523024]

25. Wang D, Bangash AK, Rhee TK, et al. Liver tumors: monitoring embolization in rabbits with VX2 tumors--transcatheter intraarterial first-pass perfusion MR imaging. Radiology 2007;245:130-139. [PubMed: 17885186]

26. Wang D, Virmani S, Tang R, et al. Four-Dimensional Transcatheter Intraarterial Perfusion (TRIP)MRI for Monitoring Liver Tumor Embolization in VX2 Rabbits. Magnetic Resonance Imaging. 2008 (In press).

27. Kern S, Hruban R, Hollingsworth MA, et al. A white paper: the product of a pancreas cancer think tank. Cancer Res 2001;61:4923-4932. [PubMed: 11406572]

28. Grippo PJ, Sandgren EP. Modeling pancreatic cancer in animals to address specific hypotheses. Methods Mol Med 2005;103:217-243. [PubMed: 15542910]

29. Case RM. Is the rat pancreas an appropriate model of the human pancreas? Pancreatology 2006;6:180-190. [PubMed: 16534243]

30. Chen JH, Lin YC, Huang YS, Chen TJ, Lin WY, Han KW. Induction of VX2 carcinoma in rabbit liver: comparison of two inoculation methods. Lab Anim 2004;38:79-84. [PubMed: 14979992]

31. Hu J, Liu ZS, Tang SL, He YM. Effect of hydroxyapatite nanoparticles on the growth and p53/cMyc protein expression of implanted hepatic VX2 tumor in rabbits by intravenous injection. World J Gastroenterol 2007;13:2798-2802. [PubMed: 17569114]

32. Gu T, Li CX, Feng Y, Wang Q, Li CH, Li CF. Trans-arterial gene therapy for hepatocellular carcinoma in a rabbit model. World J Gastroenterol 2007;13:2113-2117. [PubMed: 17465458]

33. Mostafa EM, Ganguli S, Faintuch S, Mertyna P, Goldberg SN. Optimal strategies for combining transcatheter arterial chemoembolization and radiofrequency ablation in rabbit VX2 hepatic tumors. J Vasc Interv Radiol 2008;19:1740-1748. [PubMed: 18951042]

34. Vossen JA, Buijs M, Syed L, et al. Development of a new orthotopic animal model of metastatic liver cancer in the rabbit VX2 model: effect on metastases after partial hepatectomy, intra-arterial treatment with 3-bromopyruvate and chemoembolization. Clin Exp Metastasis 2008;25:811-817. [PubMed: 18649116]

35. Rosi NL, Giljohann DA, Thaxton CS, Lytton-Jean AK, Han MS, Mirkin CA. Oligonucleotidemodified gold nanoparticles for intracellular gene regulation. Science 2006;312:1027-1030. [PubMed: 16709779]

36. Imai K, Hirata S, Irie A, et al. Identification of a novel tumor-associated antigen, cadherin 3/Pcadherin, as a possible target for immunotherapy of pancreatic, gastric, and colorectal cancers. Clin Cancer Res 2008;14:6487-6495. [PubMed: 18927288]

37. Barnett BP, Arepally A, Karmarkar PV, et al. Magnetic resonance-guided, real-time targeted delivery and imaging of magnetocapsules immunoprotecting pancreatic islet cells. Nat Med 2007;13:986-991. [PubMed: 17660829] 


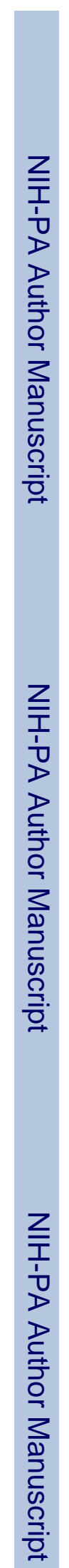

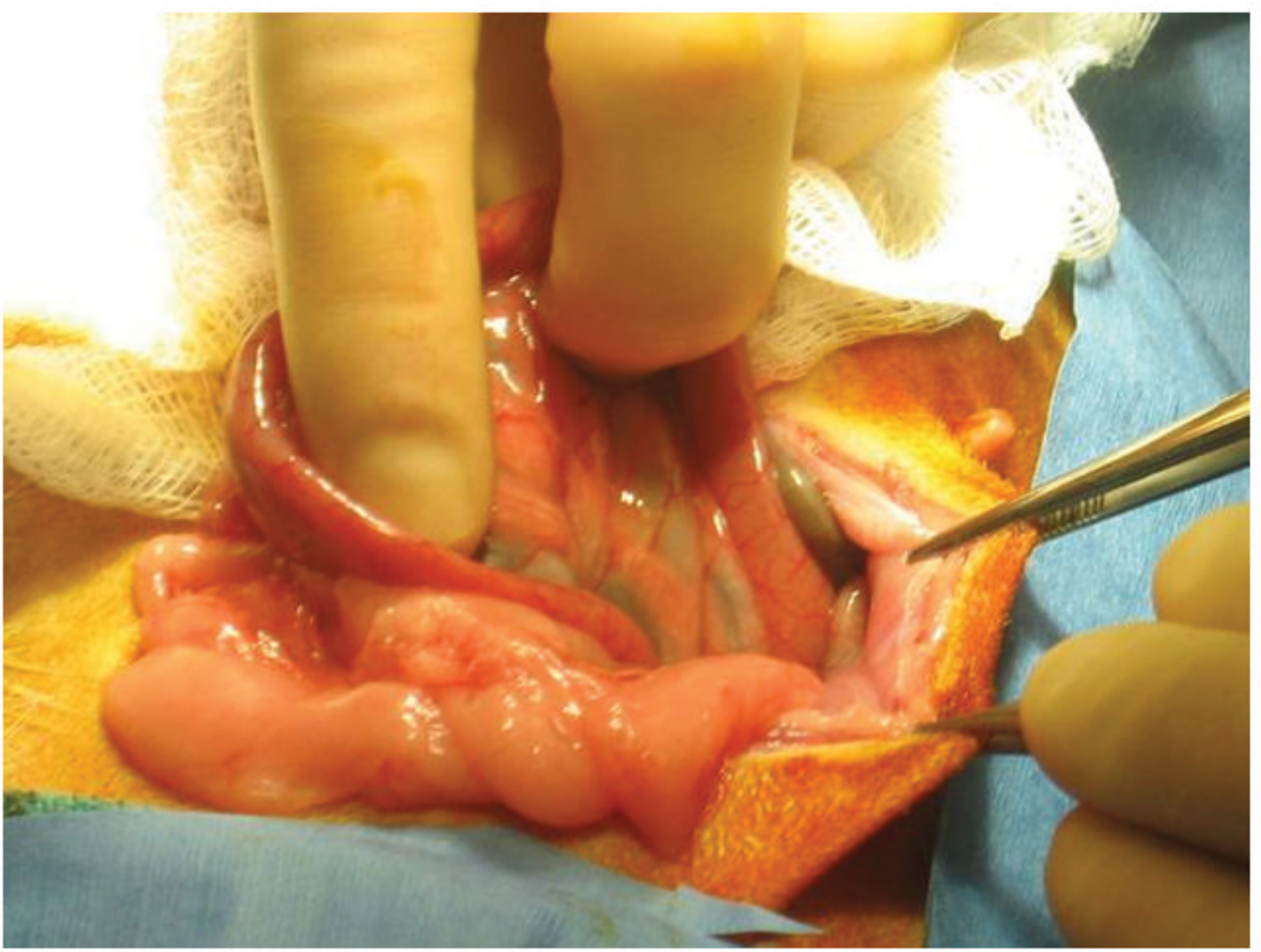

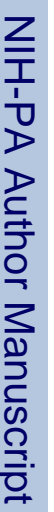

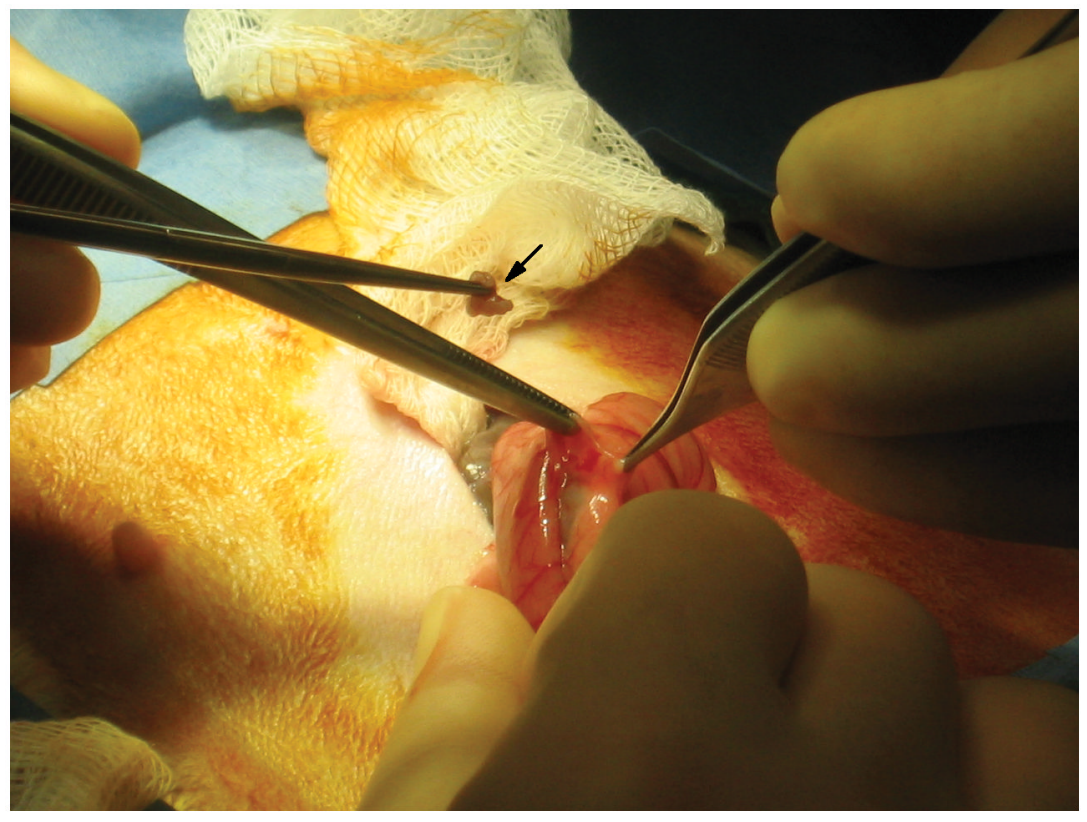




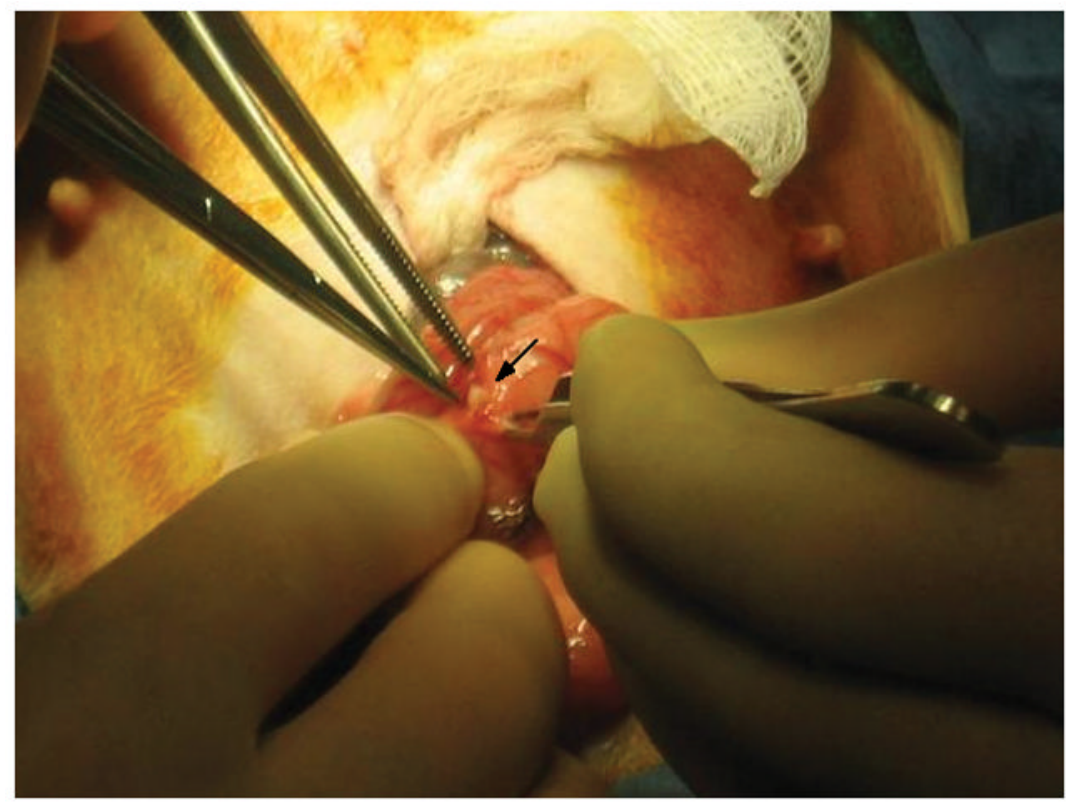

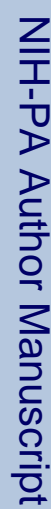

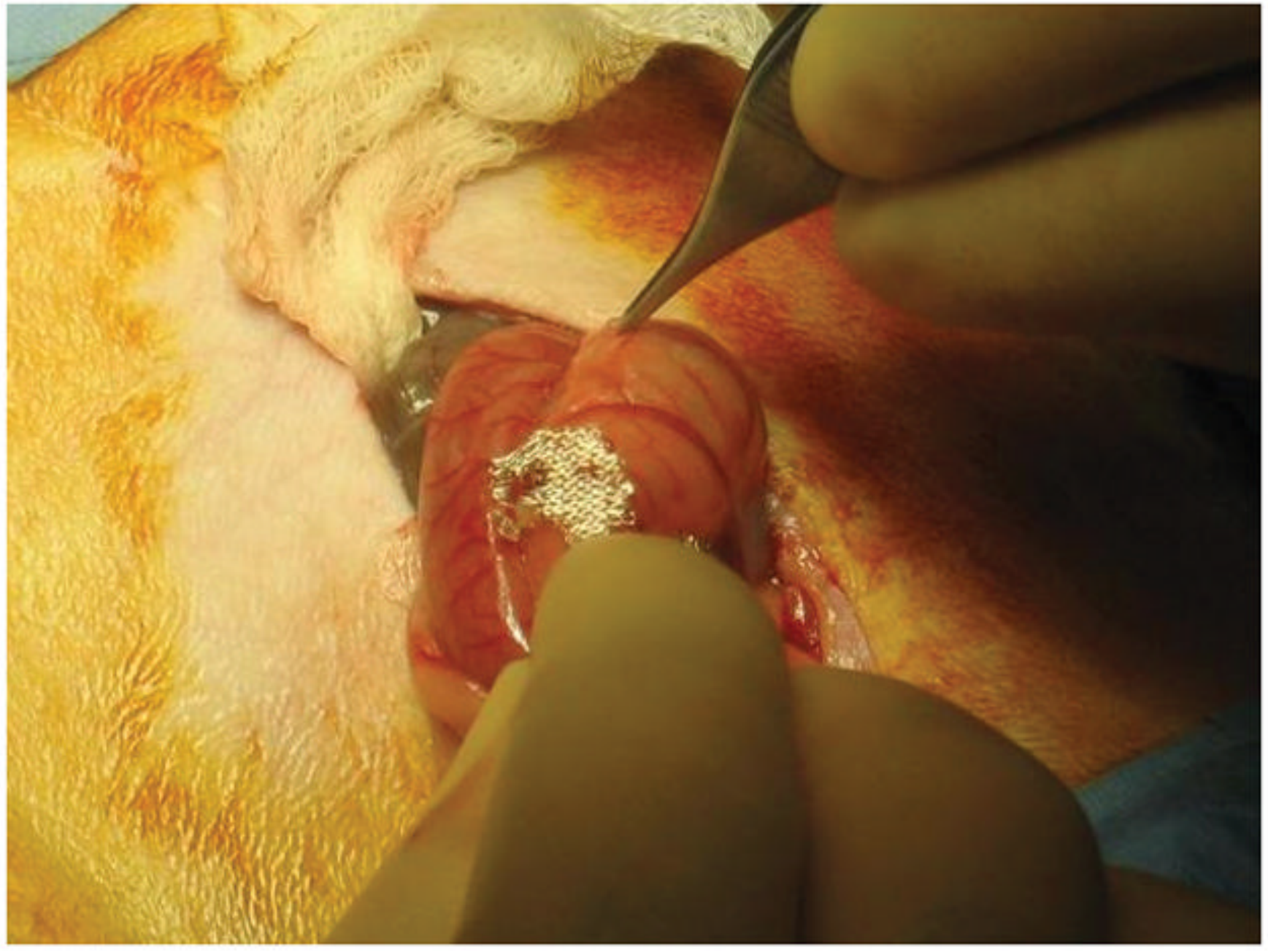


Figure 1.

The chunk implantation method for creating pancreatic tumors. (a) The pancreas is identified then exposed extracorporeally. (b, c) An incision is made in the pancreatic tissue and a small tumor piece (arrow) is inserted. (d) The incision, with tumor cells inside, is sealed with Surgicel (absorbable hemostat) and replaced into the abdomen. 


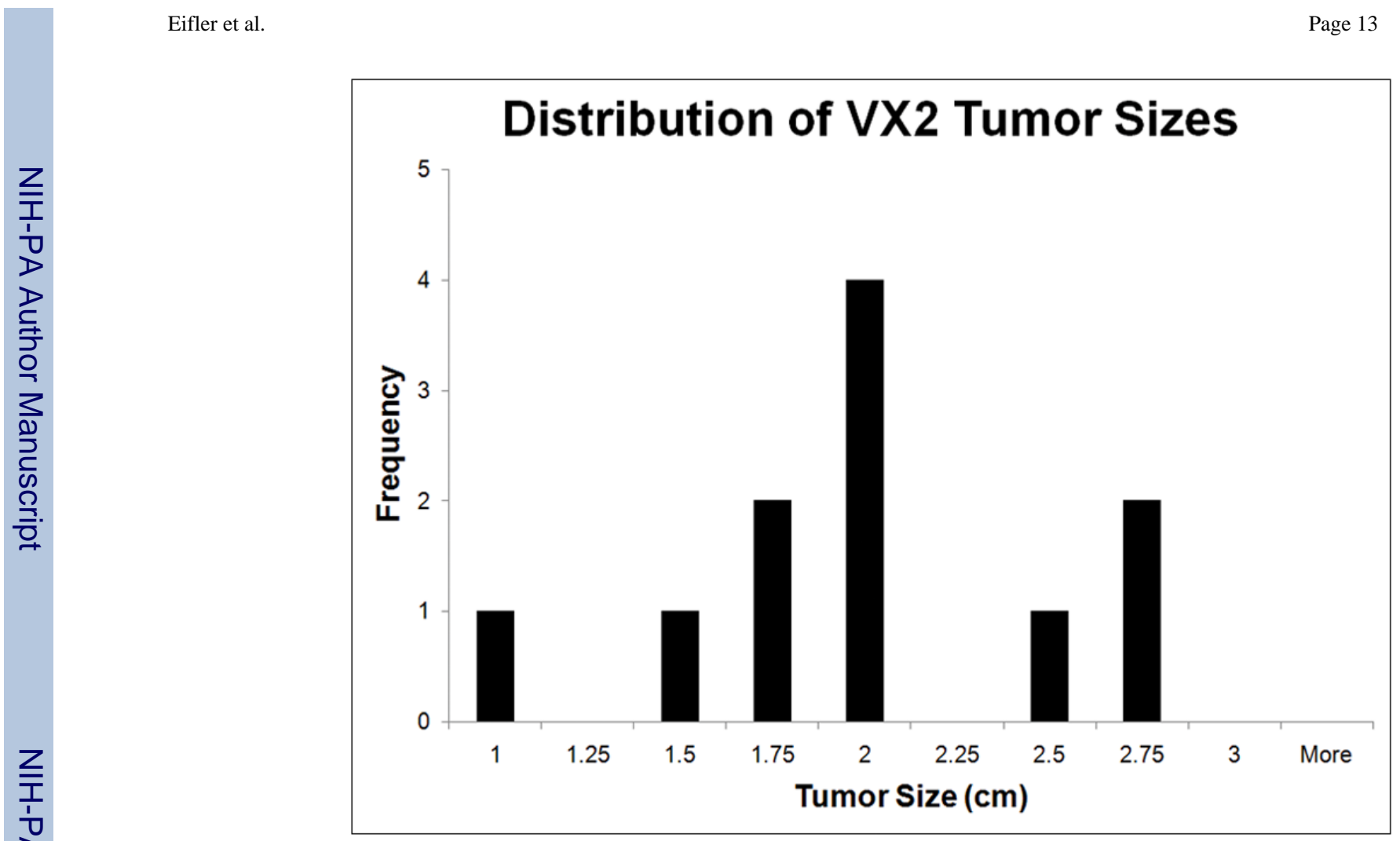

Figure 2.

Histogram showing the frequency of different tumor sizes at 3 weeks. 


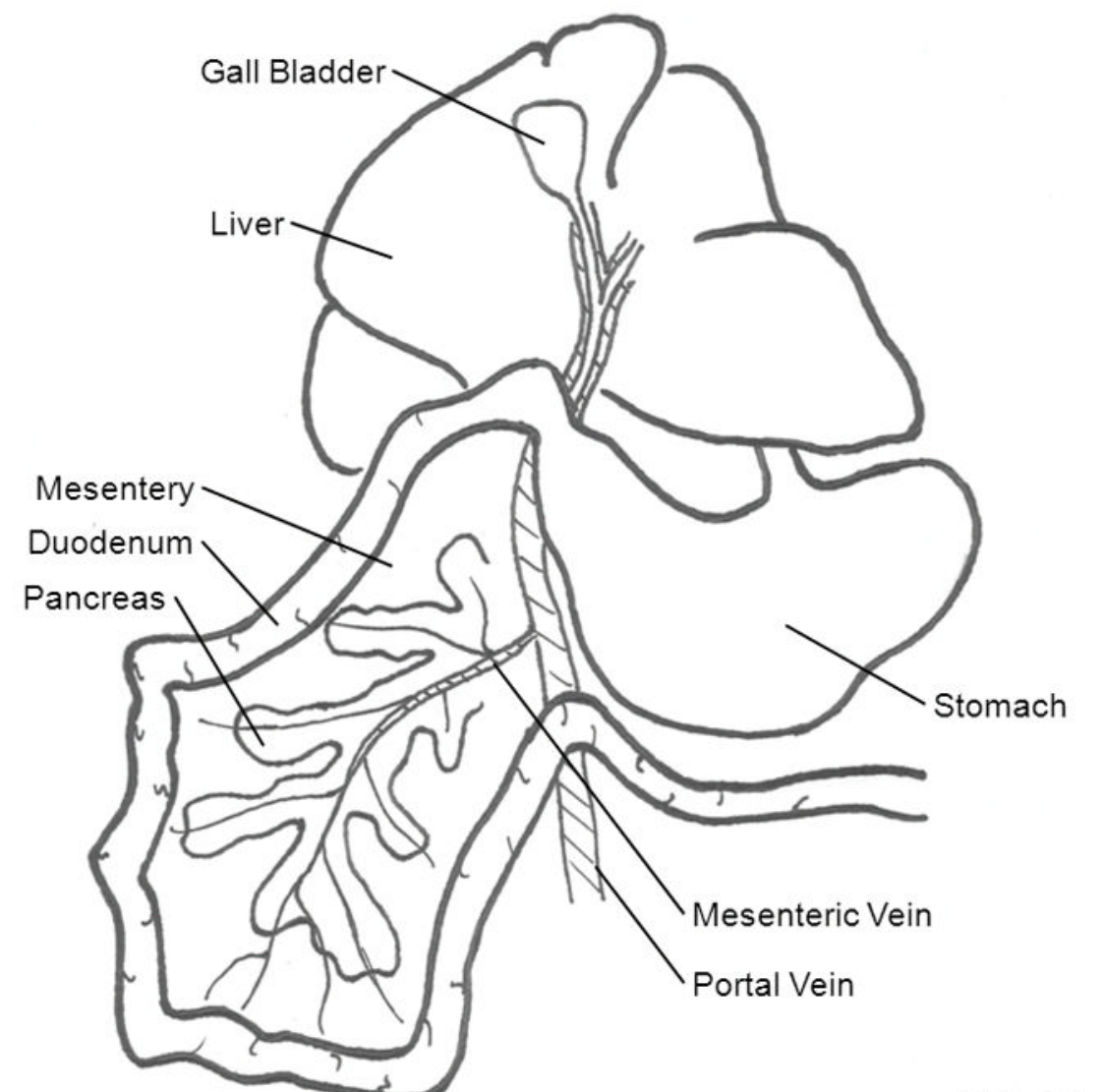

AC Eifler 2008

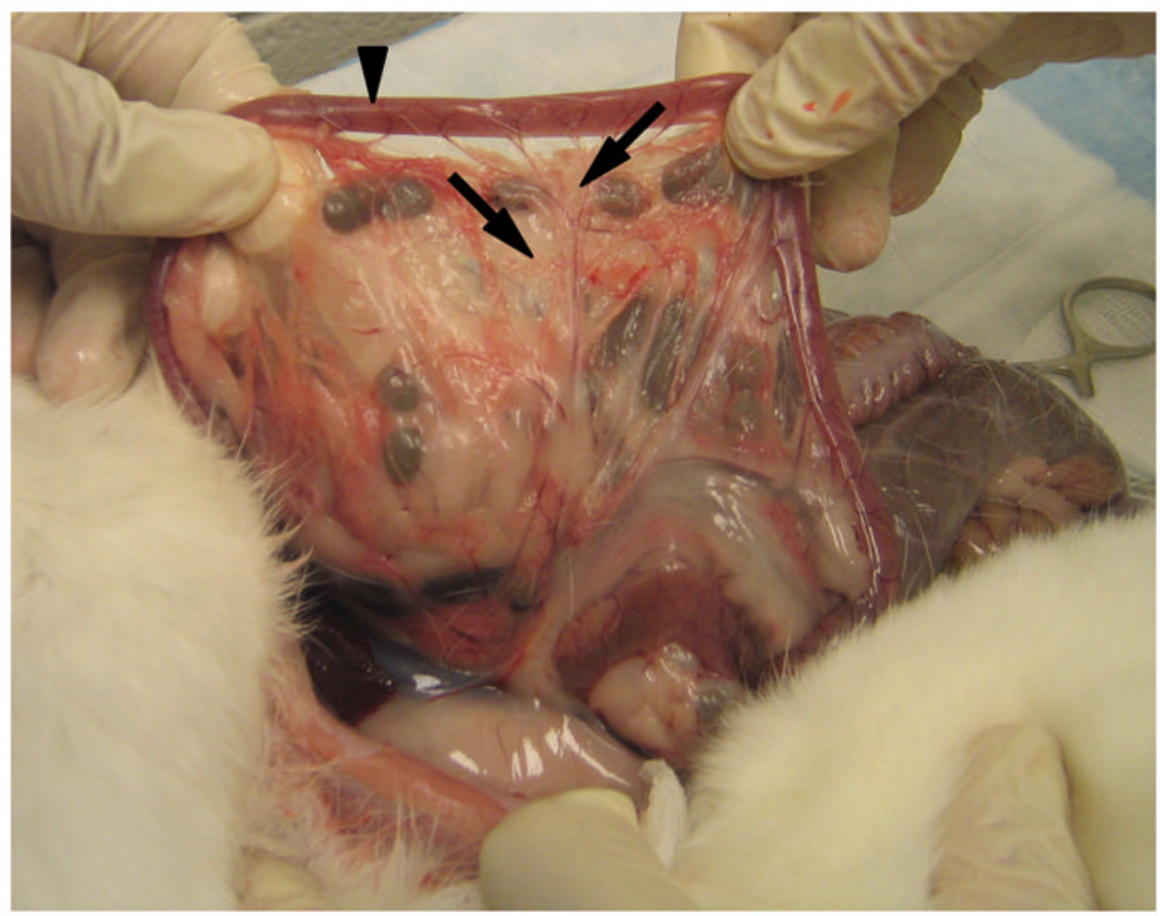




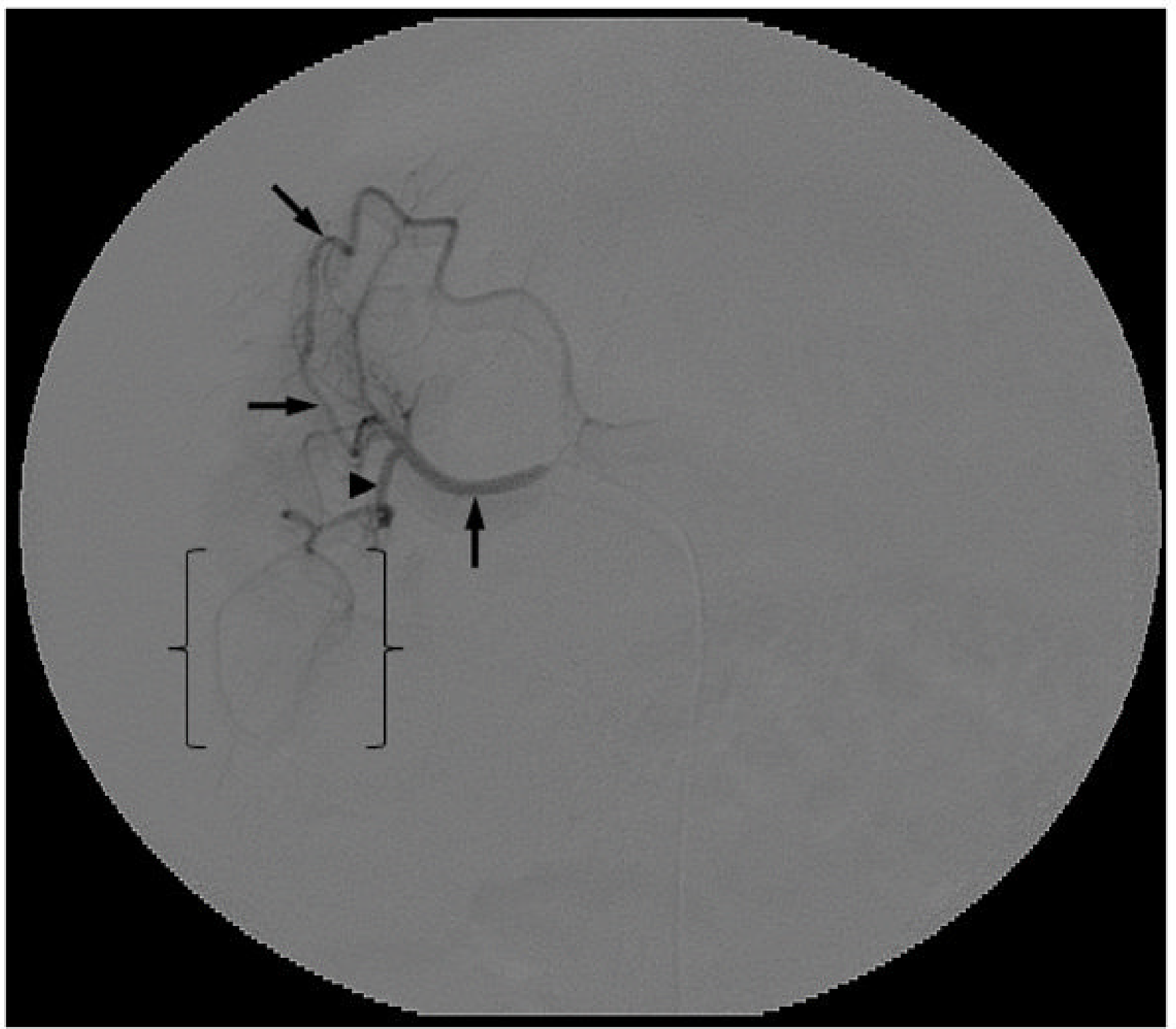

Figure 3.

Anatomy of rabbit pancreas and vasculature. (a) Diagram shows the rabbit pancreatic anatomy. (b) Photograph of sacrificed hindlimb donor rabbit showing diffuse pancreatic tissue (arrows) spread throughout the mesentery that suspends the duodenal C-loop (arrowhead). (c) DSA image of the gastroduodenal artery (arrows) showing the pancreaticoduodenal arcade (arrowhead) supplying a pancreatic tumor (brackets). 

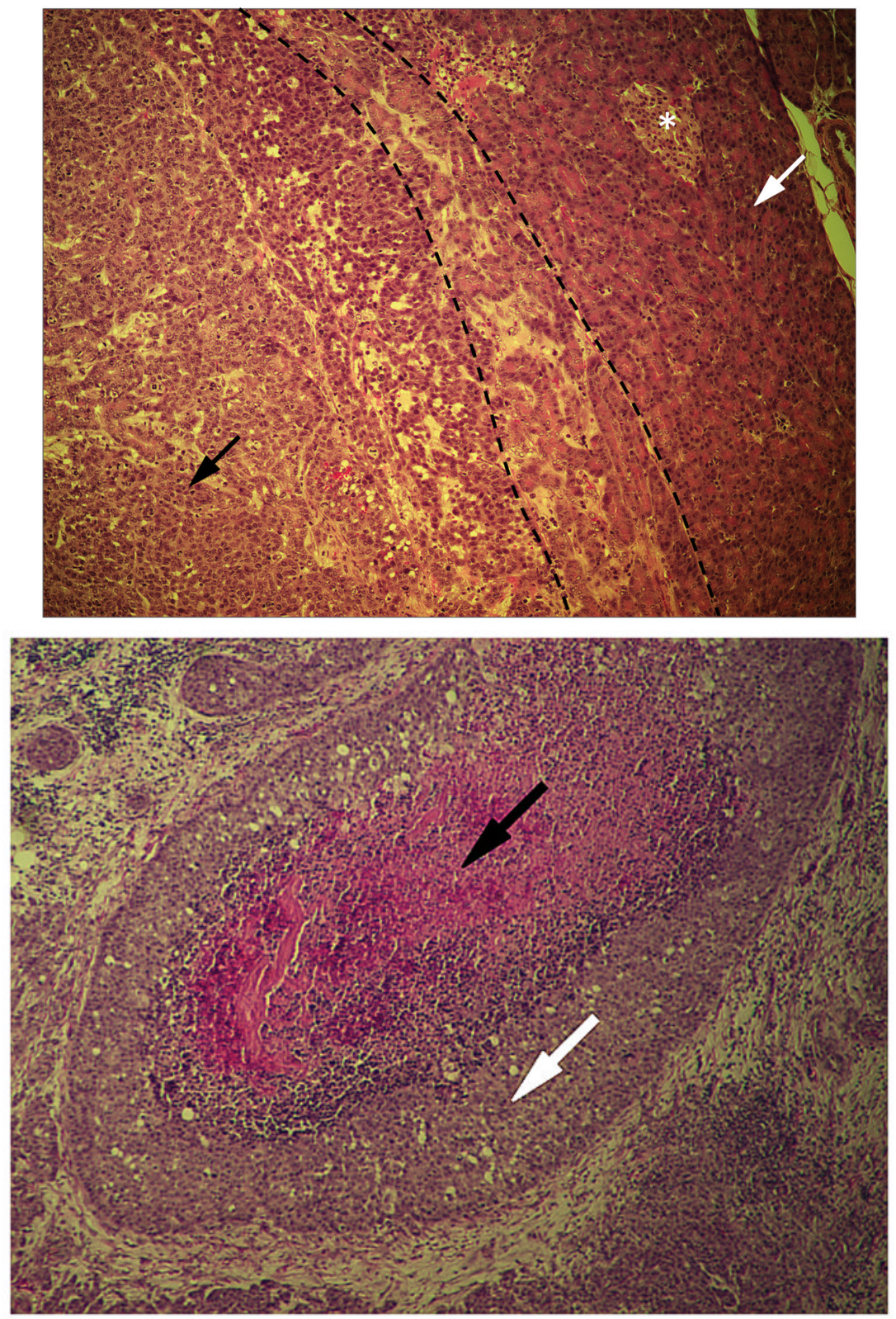


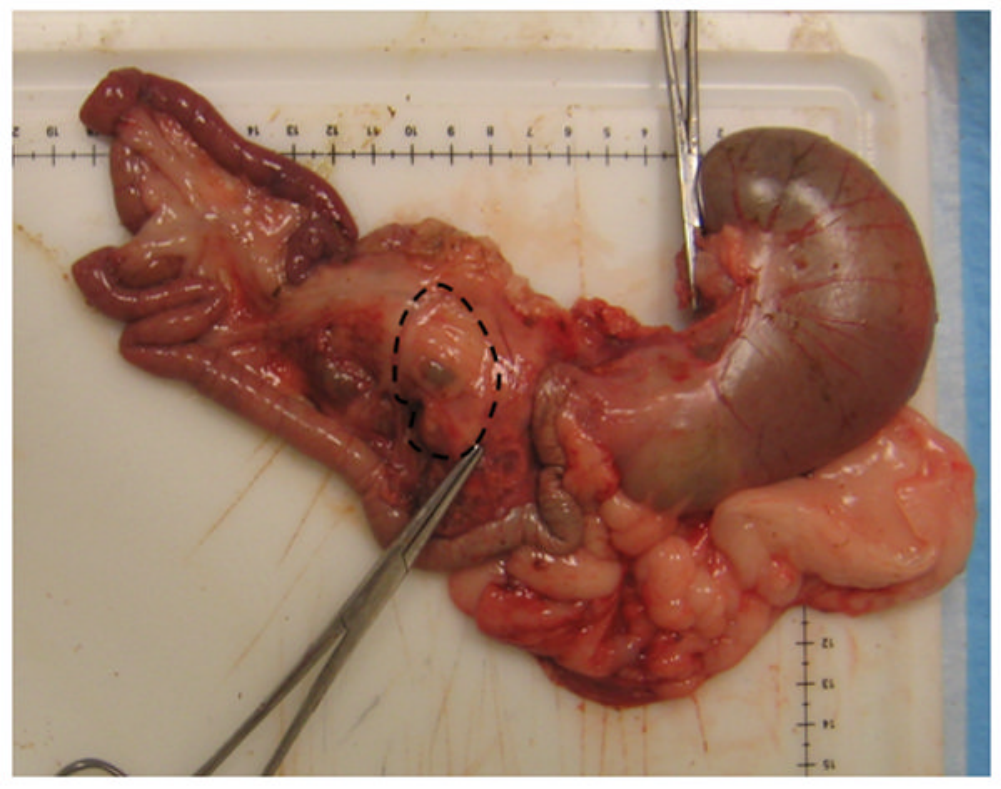

Figure 4.

Gross and histopathologic images acquired at necropsy. (a) Histopathologic image obtained at necropsy (hematoxylin-eosin stain; original magnification X25) demonstrates border between viable VX2 tumor cells (black arrow) and healthy pancreatic tissue (white arrow) with irritated pancreatitis-like tissue at the tumor-pancreas interface (between dashed lines). Note islet of Langerhans (asterisk) within healthy pancreatic tissue. (b) Histopathologic image showing VX2 tumor lobule with typical distribution of necrotic tissue (black arrow) located in the tumor core with surrounding viable tissue (white arrow) at the tumor periphery (hematoxylin-eosin stain; original magnification X12.5). (c) Photograph of pancreatic tumor (inside dashed line). Scale is in centimeters. 


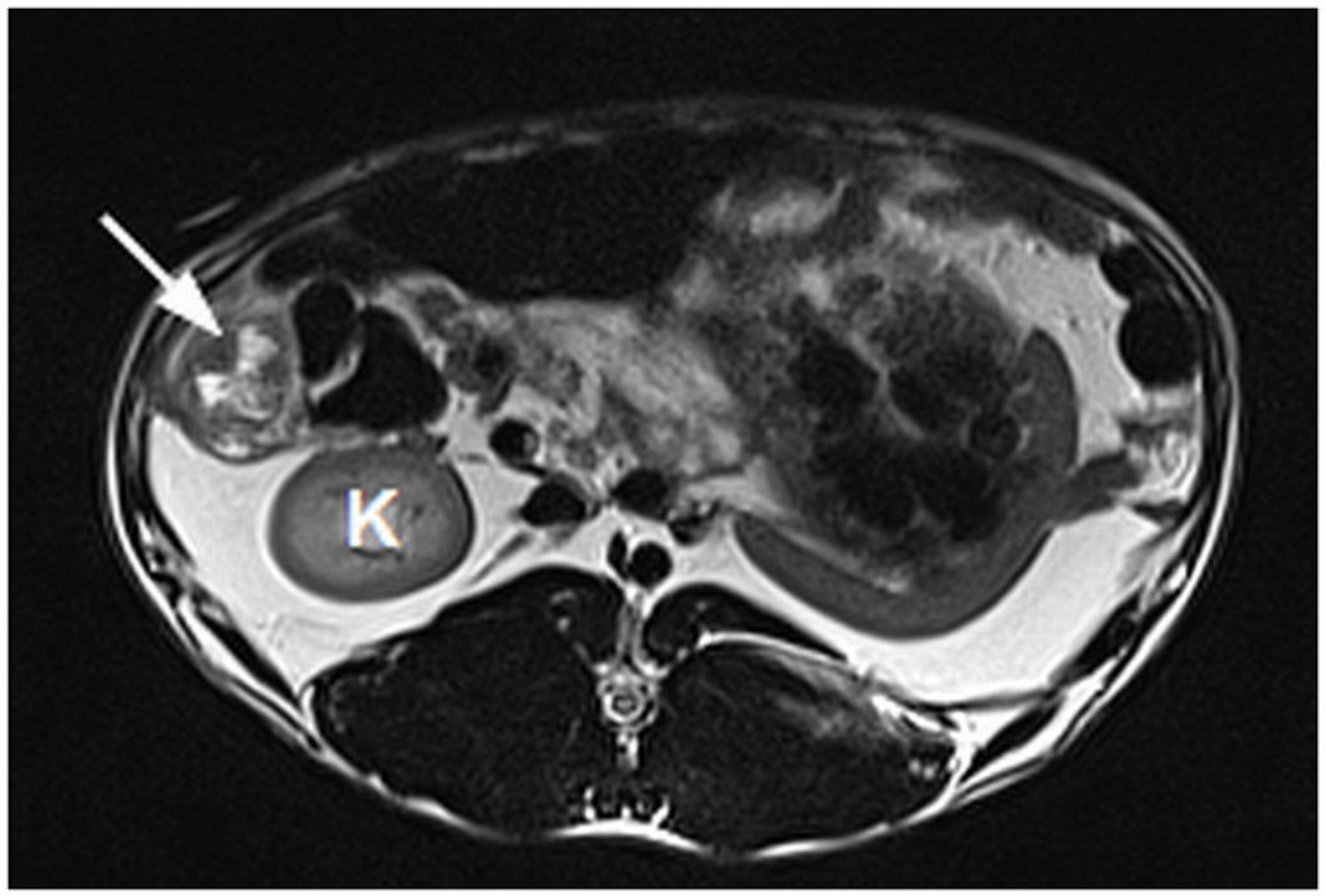




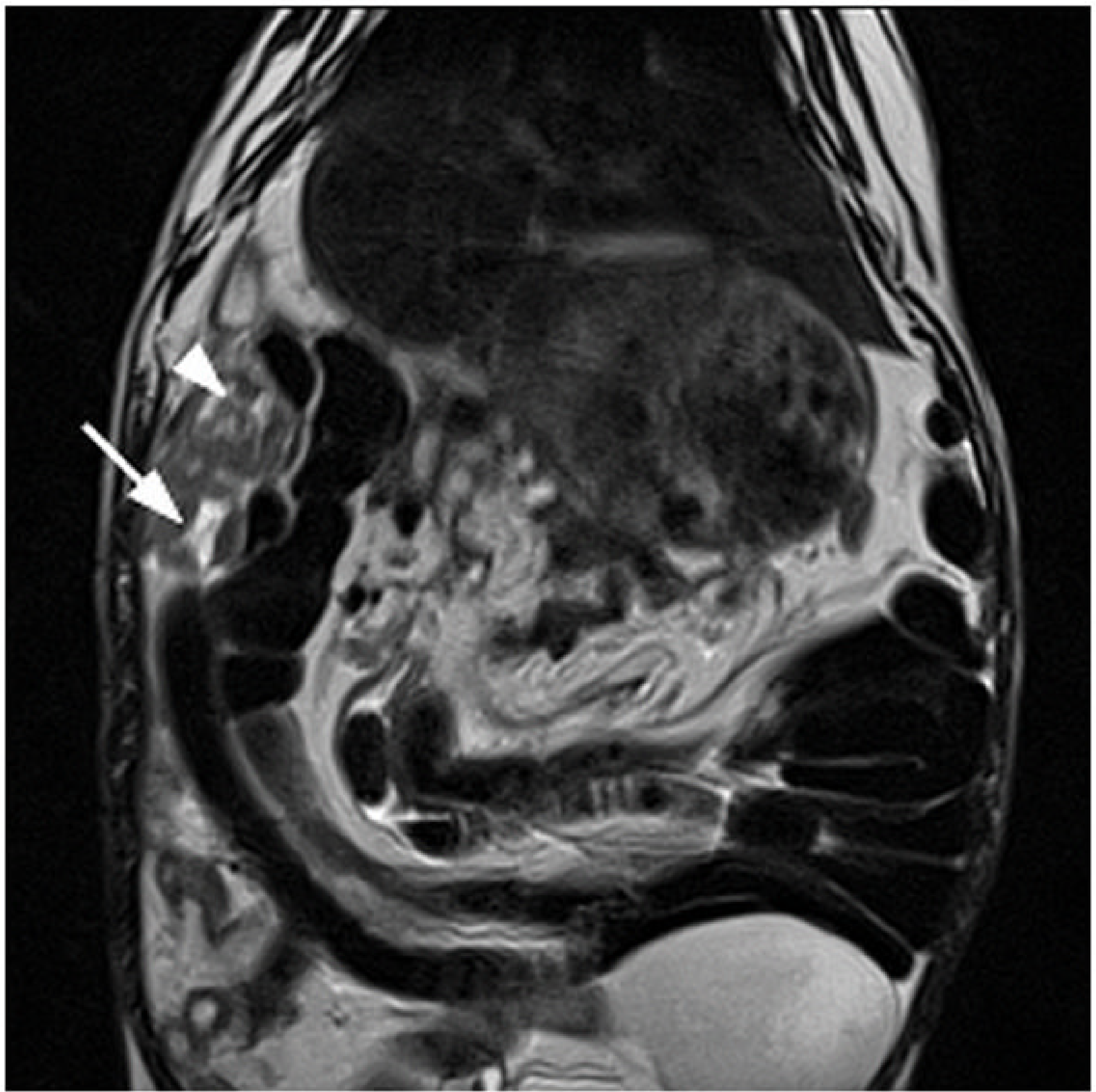

Figure 5.

Anatomic MR images of rabbit abdomen. (a) T2-weighted turbo spin echo MR images showing pancreatic tumor (arrow) in axial view. Kidney is also shown (K). (b) T2-weighted MR image shows a coronal view of the same pancreatic tumor (arrow) with second tumor (arrowhead) not visible in axial view. 


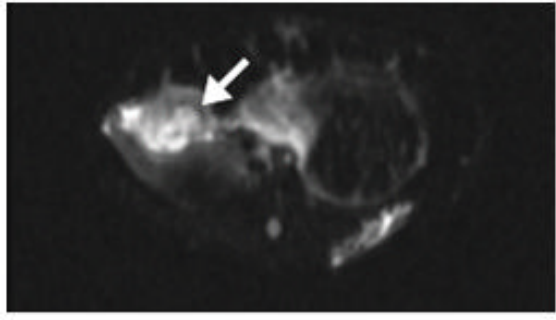

$b=0$

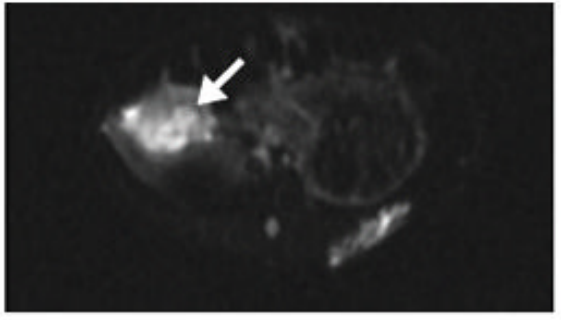

$b=50$

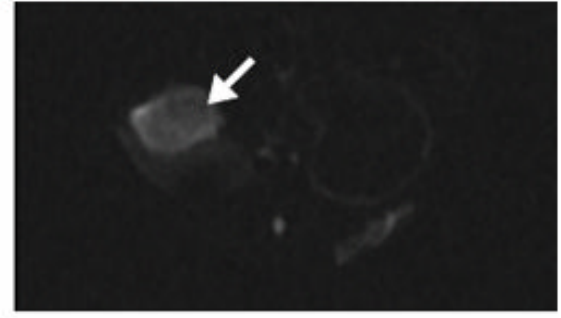

$b=500$

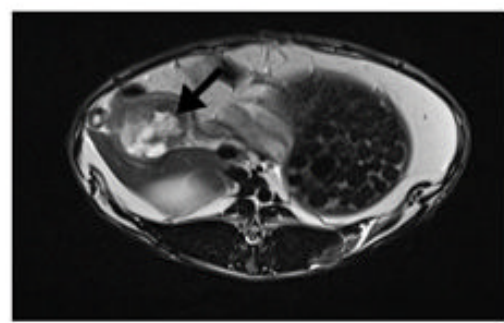

T2W TSE

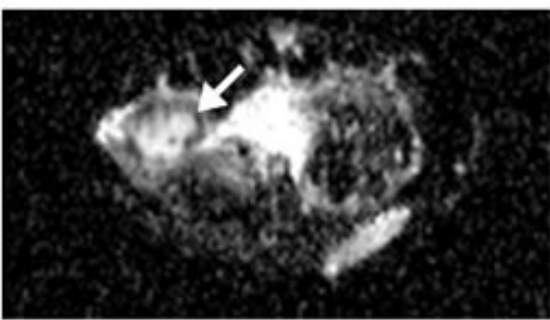

ADC Map

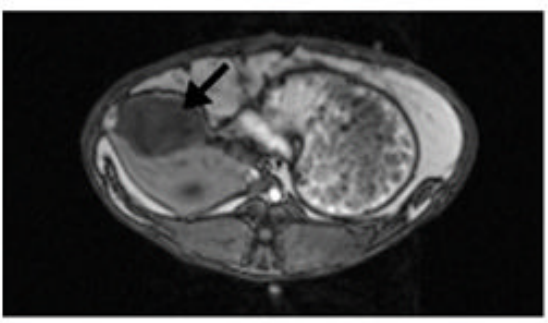

Pre-TRIP

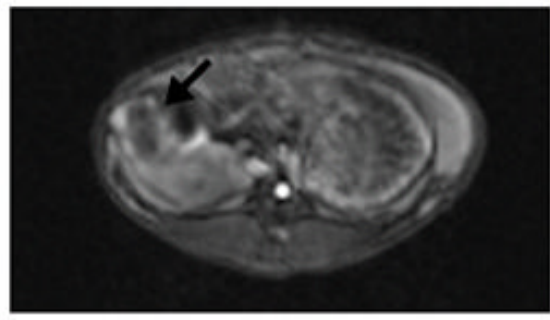

TRIP

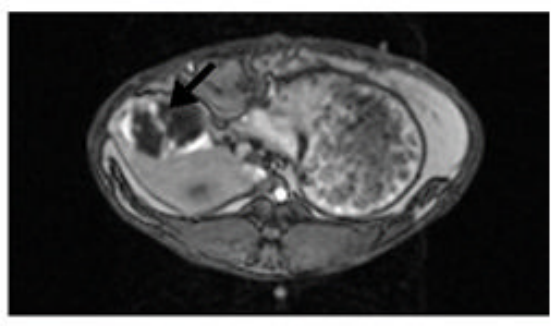

Post-TRIP

Figure 6.

Representative figure of VX2 pancreatic tumor imaged with single shot spin-echo echo planar diffusion weighted MR $\left(b=0,50,500 \mathrm{sec} / \mathrm{mm}^{2}\right)$ with corresponding ADC map, T2weighted turbo spin echo MR, T1-weighted spoiled gradient echo MR taken at 1.6 second intervals during contrast injection (TRIP MR), and T1-weighted gradient-echo MR (taken before and after TRIP imaging occurred). Each image shows a VX2 tumor (solid arrows) located in the pancreas. Note the relatively larger ADC value in the necrotic tumor core when compared to the viable periphery. This viable periphery corresponds to areas of increased perfusion as shown in the TRIP image. Additionally, this tumor is likely a conglomerate of two implanted tumor pieces as shown by the TRIP and post-TRIP MR images. 